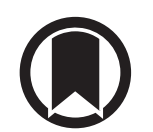

CrossMark

\title{
Unresolved issues in treatment outcome definitions for nontuberculous mycobacterial pulmonary disease
}

\author{
To the Editor:
}

The incidence and prevalence of nontuberculous mycobacterial pulmonary disease (NTM-PD) is increasing worldwide $[1,2]$. NTM-PD treatment is challenging due to long-term, multiple antibiotic therapy and poor treatment outcomes [3,4]. Standardised treatment outcome definitions for NTM-PD were recently reported in the Nontuberculous Mycobacteria Network European Trials Group (NTM-NET) consensus statement [5], and are an important development for patient management and clinical research [5].

The NTM-NET consensus statement discussed some unresolved issues in NTM-PD treatment outcomes, such as optimal timing for declaring treatment failure [5]. In the NTM-NET statement, treatment failure was considered to occur after $\geqslant 12$ months of unsuccessful treatment [5]. Assessment of treatment failure after $\geqslant 12$ months of treatment was also recommended in current NTM guidelines [6,7]. Although a recent study suggested that a lack of microbiological response after 6 months of treatment was an accurate predictor of treatment failure at 12 months [8], and a 6-month time point was used to this effect in recent clinical trials $[9,10]$, the correlation between microbiological responses at 6 months and 12 months needs further evaluation. In addition, in the NTM-NET statement, a single positive culture after culture conversion during antibiotic treatment was not regarded as treatment failure [5]. However, the clinical significance of such a single positive culture has not been systemically evaluated in the literature.

The objective of this study was to evaluate the correlation between the microbiological responses at 6 months and 12 months of treatment and to investigate the clinical significance of a single positive culture after culture conversion during antibiotic treatment in our cohort of patients with Mycobacterium avium complex (MAC)-PD.

Using our previously published data [11], we performed a post hoc analysis. The study population comprised 481 patients with treatment-naïv MAC-PD who started antibiotic treatment between January 2002 and December 2013. In that study, sputum culture conversion (SCC) was defined as three consecutive negative cultures, and the time to SCC was determined based on the date of the first negative culture [11]. Patients who failed SCC by 12 months of treatment, but finally achieved such conversion after 12 months of treatment, while on continuing medical treatment or after surgical resection, were included under the "favourable outcomes" group in that study [11].

Of the 481 patients in the original study, 470 patients were included in the current study, after excluding 11 patients who underwent surgical treatment within 12 months of treatment. For this study, we re-evaluated the numbers of patients who achieved SCC within six or 12 months of treatment. Among 470 patients, 357 (76\%) patients achieved and 113 (24\%) failed SCC within 12 months of treatment (figure 1a). Of the 357 patients who achieved SCC within 12 months, 336 (94\%) achieved this conversion within 6 months, and 21 (6\%) achieved conversion between six and 12 months of treatment. Among the 113 patients without SCC within 12 months, 105 (93\%) failed to achieve conversion within 6 months of treatment, and eight (7\%) initially achieved conversion within 6 months (median 1.8 months, interquartile range 1.1-2.7 months), but then had re-emergence of multiple positive cultures of the causative species.

@ERSpublications

Evaluation of treatment outcomes after 6 months may be appropriate for NTM pulmonary disease (NTM-PD) patients. Patients with a single positive culture after culture conversion should be carefully followed up to detect recurrence of NTM-PD. http://ow.ly/JrSy30oj3uZ

Cite this article as: Moon SM, Jhun BW, Daley CL, et al. Unresolved issues in treatment outcome definitions for nontuberculous mycobacterial pulmonary disease. Eur Respir J 2019; 53: 1801636 [https:// doi.org/10.1183/13993003.01636-2018]. 
a)
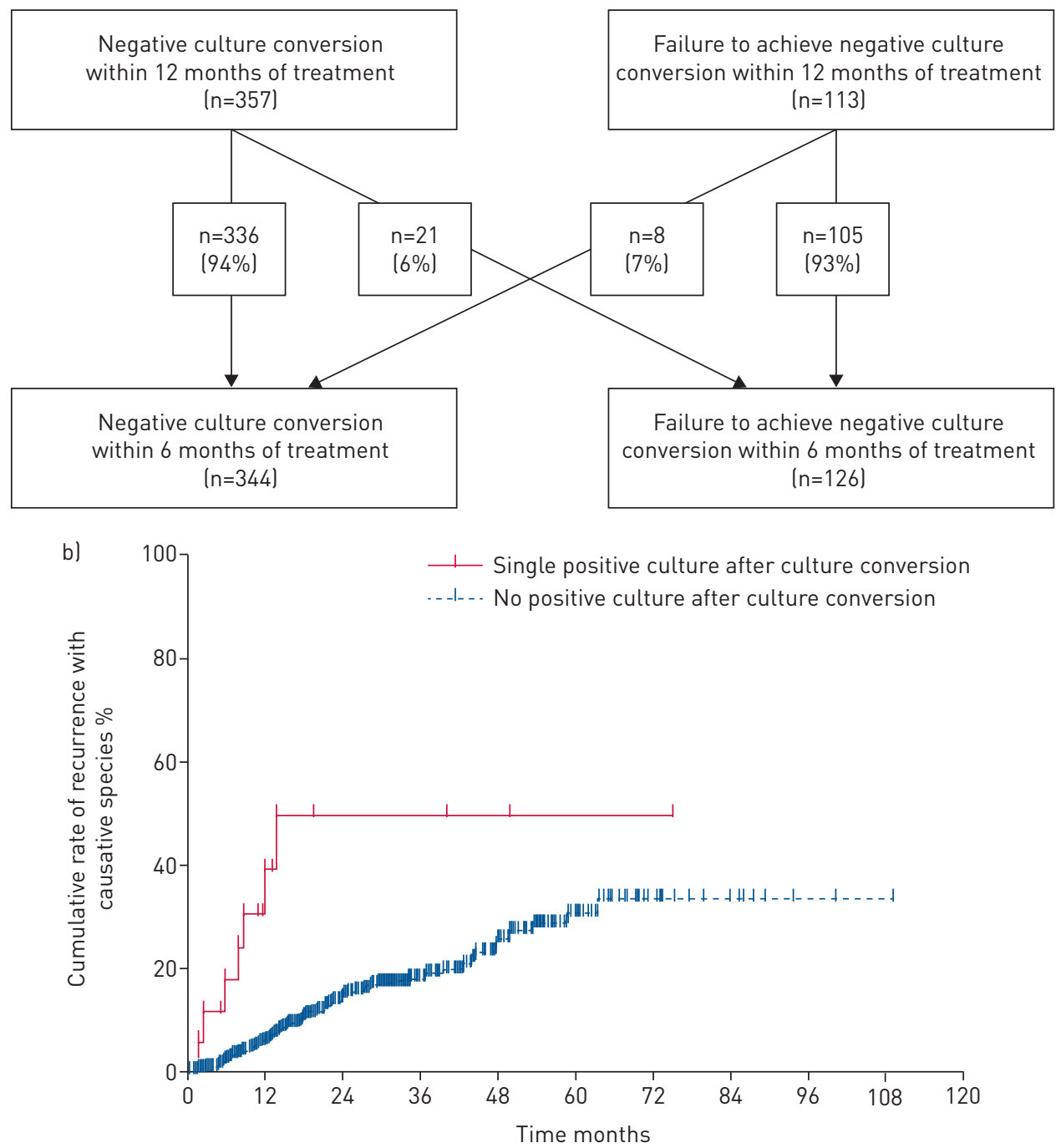

FIGURE 1 a) Treatment outcomes of 470 patients with Mycobacterium avium complex pulmonary disease. b) Cumulative rates of recurrence with causative species after treatment completion of $M$. avium complex pulmonary disease.

To investigate the clinical significance of a single positive culture after SCC during antibiotic treatment, the risk of recurrence with the causative species after treatment completion was evaluated. A single positive culture was counted if the causative species was cultured only once between SCC and the end of treatment.

Among the 402 patients with final favourable outcomes in our previous study, 377 (94\%) maintained negative cultures after SCC until the end of treatment, and $25(6 \%)$ had a single positive culture of the causative species after SCC during treatment. For these two sets of patients, recurrence of MAC-PD was defined as the re-emergence of at least two positive cultures with the same MAC species after treatment completion [5]. The Kaplan-Meier method was used to estimate the cumulative rates of recurrence involving the causative species, and the log-rank test was applied to compare the curves for the two patient sets (figure $1 \mathrm{~b}$ ). Cox regression was used to evaluate the hazard ratio of a single positive culture on recurrence with the causative species. The data were analysed using SPSS software (version 23; IBM Corp., Armonk, NY, USA) and GraphPad Prism software (GraphPad Software, La Jolla, CA, USA). At 12 months, 36 months and 60 months from treatment completion, respectively, the cumulative rates of recurrence of MAC-PD with causative species were $39 \%, 50 \%$ and $50 \%$ in patients with a single positive culture after SCC and 7\%, 19\% and 32\% in patients with no positive culture after SCC. These recurrence rates were significantly different between the two groups (log-rank test: p-value $=0.001$; hazard ratio 3.18 (95\% CI 1.51-6.69)) (figure 1b). 
Using nearly 500 previously studied MAC-PD cases, this study evaluated the correlation between the microbiological responses at six and 12 months of treatment and investigated the clinical significance of a single positive culture after SCC during antibiotic treatment. We found a concordance rate of $>90 \%$ in the microbiological responses at six and 12 months of treatment. In addition, patients who had a single positive culture after SCC during treatment were more likely to experience recurrence with the causative species after treatment completion.

Although previous guidelines and the NTM-NET consensus statement recommend that treatment outcomes be evaluated after 12 months of treatment [5-7], recent studies evaluating new or repurposed drugs for NTM-PD considered the definition of refractory NTM-PD to involve a lack of microbiological response at 6 months of treatment $[9,10,12-14]$. The optimal timing for decision of treatment failure must balance premature discontinuation of an effective treatment regimen and prolonged administration of an ineffective regimen. A previous study revealed that $83 \%$ of MAC-PD patients who showed SCC had their first negative culture within 6 months of treatment [8], consistent with our findings. Therefore, the evaluation of treatment outcomes might be more appropriate after confirmation of sputum culture results obtained at six instead of 12 months of treatment, and patients who do not achieve SCC after 6 months of treatment should be considered for further interventions including surgical resection or new or repurposed drugs.

The definition for treatment failure includes multiple positive cultures after antibiotic treatment [5]. Although a previous study showed that $13 \%$ (23 out of 180) of MAC-PD patients had a single MAC isolate after sputum conversion [15], the clinical meaning of this finding was largely unknown. Our study found that even a single positive culture after SCC to negative was associated with recurrence of MAC-PD, and therefore, such patients need to be carefully followed up. However, genotyping results for single positive cultures after SCC were not available in our study, so we could not distinguish between an original strain (relapse) and a new strain (reinfection).

In conclusion, the absence of culture conversion by 6 months of treatment may be a reliable predictor of treatment failure at 12 months of treatment. Therefore, the evaluation of treatment outcomes after 6 months may be appropriate for patient management and future clinical studies. Patients with a single positive culture after SCC should be carefully followed up to detect recurrence of NTM-PD, even following the declaration of successful treatment completion. The recommended treatment outcome definitions for NTM-PD need to be evaluated for appropriateness in different cohorts at a global level.

Seong Mi Moon ${ }^{1}$, Byung Woo Jhun $\odot^{1}$, Charles L. Daley ${ }^{2}$ and Won-Jung Koh $\oplus^{1}$

${ }^{1}$ Division of Pulmonary and Critical Care Medicine, Dept of Medicine, Samsung Medical Center, Sungkyunkwan University School of Medicine, Seoul, South Korea. ${ }^{2}$ Division of Mycobacterial and Respiratory Infections, National Jewish Health, Denver, CO, USA.

Correspondence: Won-Jung Koh, Division of Pulmonary and Critical Care Medicine, Dept of Medicine, Samsung Medical Center, Sungkyunkwan University School of Medicine, 81 Irwon-ro, Gangnam-gu, Seoul 06351, South Korea. E-mail:wjkoh@skku.edu

Received: Aug 282018 | Accepted after revision: Feb 132019

Author Contributions: Study conception and design: Seong Mi Moon, Byung Woo Jhun and Won-Jung Koh. Data acquisition and analysis: Seong Mi Moon and Byung Woo Jhun. Data interpretation and manuscript writing: Seong Mi Moon and Won-Jung Koh. Critical revision and final approval of the manuscript: Seong Mi Moon, Byung Woo Jhun, Charles L. Daley and Won-Jung Koh.

Conflict of interest: S.M. Moon has nothing to disclose. B.W. Jhun has nothing to disclose. C.L. Daley reports grants and personal fees from Insmed, and personal fees from Horizon, Spero and Johnson and Johnson, outside the submitted work. W-J. Koh has received a consultation fee from Insmed Inc. for the Insmed Advisory Board Meeting, outside the submitted work.

Support statement: This research was supported by the National Research Foundation of Korea (NRF) funded by the Korea government (MSIT) (NRF-2018R1A2A1A05018309). The sponsor had no role in the design of the study, the collection and analysis of the data, or the preparation of the manuscript. Funding information for this article has been deposited with the Crossref Funder Registry.

\section{References}

1 Prevots DR, Marras TK. Epidemiology of human pulmonary infection with nontuberculous mycobacteria: a review. Clin Chest Med 2015; 36: 13-34.

2 Adjemian J, Daniel-Wayman S, Ricotta E, et al. Epidemiology of nontuberculous mycobacteriosis. Semin Respir Crit Care Med 2018; 39: 325-335.

3 Kwak N, Park J, Kim E, et al. Treatment outcomes of Mycobacterium avium complex lung disease: a systematic review and meta-analysis. Clin Infect Dis 2017; 65: 1077-1084. 
Diel R, Nienhaus A, Ringshausen FC, et al. Microbiologic outcome of interventions against Mycobacterium avium complex pulmonary disease: a systematic review. Chest 2018; 153: 888-921.

5 van Ingen J, Aksamit T, Andrejak C, et al. Treatment outcome definitions in nontuberculous mycobacterial pulmonary disease: an NTM-NET consensus statement. Eur Respir J 2018; 51: 1800170.

6 Griffith DE, Aksamit T, Brown-Elliott BA, et al. An official ATS/IDSA statement: diagnosis, treatment, and prevention of nontuberculous mycobacterial diseases. Am J Respir Crit Care Med 2007; 175: 367-416.

7 Haworth CS, Banks J, Capstick T, et al. British Thoracic Society guidelines for the management of non-tuberculous mycobacterial pulmonary disease (NTM-PD). Thorax 2017; 72: Suppl. 2, ii1-ii64.

8 Griffith DE, Adjemian J, Brown-Elliott BA, et al. Semiquantitative culture analysis during therapy for Mycobacterium avium complex lung disease. Am J Respir Crit Care Med 2015; 192: 754-760.

9 Olivier KN, Griffith DE, Eagle G, et al. Randomized trial of liposomal amikacin for inhalation in nontuberculous mycobacterial lung disease. Am J Respir Crit Care Med 2017; 195: 814-823.

10 Griffith DE, Eagle G, Thomson R, et al. Amikacin liposome inhalation suspension for treatment-refractory lung disease caused by Mycobacterium avium complex (CONVERT): a prospective, open-label, randomized study. Am J Respir Crit Care Med 2018; 198: 1559-1569.

11 Koh WJ, Moon SM, Kim SY, et al. Outcomes of Mycobacterium avium complex lung disease based on clinical phenotype. Eur Respir J 2017; 50: 1602503.

12 Jhun BW, Yang B, Moon SM, et al. Amikacin inhalation as salvage therapy for refractory nontuberculous mycobacterial lung disease. Antimicrob Agents Chemother 2018; 62: e00011.

13 Yang B, Jhun BW, Moon SM, et al. A clofazimine-containing regimen for the treatment of Mycobacterium abscessus lung disease. Antimicrob Agents Chemother 2017; 61: e02052.

14 Philley JV, Wallace RJ Jr, Benwill JL, et al. Preliminary results of bedaquiline as salvage therapy for patients with nontuberculous mycobacterial lung disease. Chest 2015; 148: 499-506.

15 Wallace RJ Jr, Brown-Elliott BA, McNulty S, et al. Macrolide/azalide therapy for nodular/bronchiectatic Mycobacterium avium complex lung disease. Chest 2014; 146: 276-282. 